\title{
2D and 3D Electrospun Silk Fibroin Gelatin Coatings to Improve Scaffold Performances in Cardiovascular Applications
}

\author{
Maria Cristina Tanzi *, Chiara Marcolin, Lorenza Draghi * and Silvia Farè * \\ Department of Chemistry, Materials and Chemical Engineering "G. Natta"; chiara.marcolin@polimi.it \\ and *INSTM Local Unit Politecnico di Milano, Piazza L. Da Vinci 32, Milano, Italy; \\ lorenza.draghi@polimi.it (L.D.); silvia.fare@polimi.it (S.F.) \\ * Correspondence: mariacristina.tanzi@polimi.it (M.C.T.);
}

Received: 27 April 2020; Accepted: 13 May 2020; Published: 14 May 2020

\begin{abstract}
A coating with biomacromolecules can improve the performance of electrospun 3D scaffolds and 2D matrices that show morphology similar to that of native ECM, but their mechanical and biological properties are often inadequate, particularly in applications in contact with blood.

In this work, a gelatin coating was applied to electrospun silk fibroin (ESF) mats and tubes intended for the regeneration of cardiovascular tissues. The crosslinking reaction used is based on a Michael-type addition in water that promotes the formation of covalent bonds between gelatin amino groups and $\beta$-carbons of N-N'-methylene bis-acrylamide (MBA). ESF mats and tubes were coated with gelatin MBA-crosslinked in situ by loading or dipping the ESF samples with the crosslinking solution, by use of static or dynamic home-made systems. SEM analysis on coated samples showed a homogeneous coating with gelatin penetrating the whole thickness of the SF matrix $\{\approx 120 \mu \mathrm{m}$ for mats and $\approx 212 \mu \mathrm{m}$ for tubes), with an increase of thickness of about $40 \%$ in wet conditions. Water uptake tests indicated for coated samples a faster and higher swelling $(1600 \%$ after 14 days) than not coated ones (500\%), due to the presence of gelatin.

Tensile mechanical tests showed higher values of ultimate stress and elastic modulus for silk fibroin samples $\left(\sigma_{b}=2.4, \mathrm{E}=1.82 \mathrm{MPa}\right)$ compared to gelatin-coated ones $\left(\sigma_{b}=1.2, \mathrm{E}=0.58 \mathrm{MPa}\right)$, with not significant differences in the ultimate deformation $(\approx 150 \%)$.

Indirect cytocompatibility tests, performed by culturing L929 cells in the presence of eluates obtained by immersing coated and uncoated samples up to 7 days in culture medium, demonstrated a cell viability higher than the control. For direct contact tests, primary human umbilical vein endothelial cell (HUVEC), obtained by enzymatic digestion, and cell adhesion and growth on the MBA-crosslinked gelatin was analysed by OM after fixing with formalin and staining with toluidine blue. HUVEC were seeded onto ESF and ESF-coated samples and cultured under standard tissue culture conditions. After 7 days from seeding, cell proliferation was evaluated from the quantitation of total proteins in cell lysates (BCA protein assay) and the obtained results indicated a significantly higher $(\mathrm{p}<0.05)$ cell growth on gelatin-coated ESF samples.

Overall, these results point out that the described gelatin coating allows producing a structure with adequate mechanical properties for cardio-vascular applications and biological characteristics even better than those of silk fibroin.
\end{abstract}

Keywords: crosslinked gelatin; coating; electrospun silk fibroin; swelling; tensile properties; cytocompatibility; L929; primary HUVEC 


\section{Introduction}

In recent years, the electrospinning (ES) technique has received a lot of attention in the scientific field for manufacturing morphologically useful substrates for application in tissue engineering (TE). Natural fibers in ECM have nanometric or submicrometric dimension and with ES it is possible to produce fibrous matrices with nanometric fibers made of several different polymers [1-3]. Among the suitable polymers, those of natural origin are of particular interest for biomimetic approaches in TE since they offer the advantage of reduced risk for tissue toxicity or inflammatory host immune responses. In particular, silk fibroin (SF), a protein produced by lepidopters or spiders, is characterized by a highly repetitive primary sequence of amino-acids that provides a high degree of crystallinity and central mechanical properties [4,5]. These properties, joined to the excellent biocompatibility and environmental stability of SF provide great opportunity in biomedical applications. Among the various techniques useful to process silk fibroin, ES offers an interesting opportunity for producing 2D and 3D matrices with great potential for tissue regeneration and repair [6-8]. Electrospun SF was applied to the production of scaffolds for a variety of biological applications such as bone, nerve and skin tissue [9]. In some cases, however, ES scaffold produced with a single polymer may be inadequate in providing the appropriate mechanical and biological properties for regeneration of specific tissues, particularly in applications in contact with blood, e.g., in blood vessel substitutes $[10,11]$.

Hybridization [12-14] or coating with biomacromolecules [15] can improve the performance of electrospun 3D scaffolds and 2D matrices. The hybridization of electrospun SF and type I collagen was proposed to combine the advantages intrinsic in each material and minimize their drawbacks [12].

However, collagen is potentially immunogenic because of its animal origin, and it presents high costs [16]. To overcome these limitations, gelatin can be used as an alternative [17]. Gelatin is an inexpensive natural polymer derived from a partial hydrolysis of collagen, non-immunogenic, biodegradable, easy to process and biocompatible. Such a protein also has the natural cell binding motifs like arginine-glycine-aspartic acid (RGD) that is favorable for cell activities. However, gelatin is rarely used alone owing to its high weakness, and thus needs to be modified with several methods including crosslinking, grafting and blending.

Blends of silk fibroin and gelatin polymers have been electrospun to produce scaffolds for vascular tissue engineering or drug delivery systems

Electrospun SF/gelatin nanofiber mats, as blends, have already been developed and evaluated $[18,19]$, however this approach has some intrinsic limitations, as needs to stabilize gelatin by a crosslinking reaction that may alter fiber morphology alteration [19] or other stability and/or toxicity problems.

In this work, a gelatin coating was applied to electrospun silk fibroin (ESF) mats and tubes intended for the regeneration of cardiovascular tissues. The crosslinking reaction used is based on a Michael-type addition in water that promotes the formation of covalent bonds between gelatin amino groups and $\beta$-carbons of $\mathrm{N}-\mathrm{N}^{\prime}$-methylene bis-acrylamide (MBA). MBA-crosslinking was easily obtained in situ by loading or dipping the ESF samples with the crosslinking solution, by use of static or dynamic home-made systems.

Morphology, water uptake, stability and tensile properties of 2D and 3D (tubular) gelatin-coated samples were evaluated and cytocompatibility was successfully assessed with L929 and primary Human Umbilical cord Vein Endothelial Cells.

\section{Materials and Methods}

\subsection{Preparation of $2 D$ and $3 D$ Gelatin Coated ESF Samples}

Electrospun silk fibroin (ESF) plane and tubular structures were fabricated by electrospinning, with a home-made apparatus, using a $7.5 \% \mathrm{w} / \mathrm{v}$ SF solution in formic acid, a plane (plate of $10 \times 10 \mathrm{~cm}$ ) or a rotating mandrel $(\varnothing=6 \mathrm{~mm}, 1=10 \mathrm{~cm})$ stainless steel collector, with previously described 
optimized parameters [7,8]. ESF samples were treated with methanol $(>99.9 \%)$ to induce silk fibroin crystallization. Images of the ESF structures are presented in Figure 1.

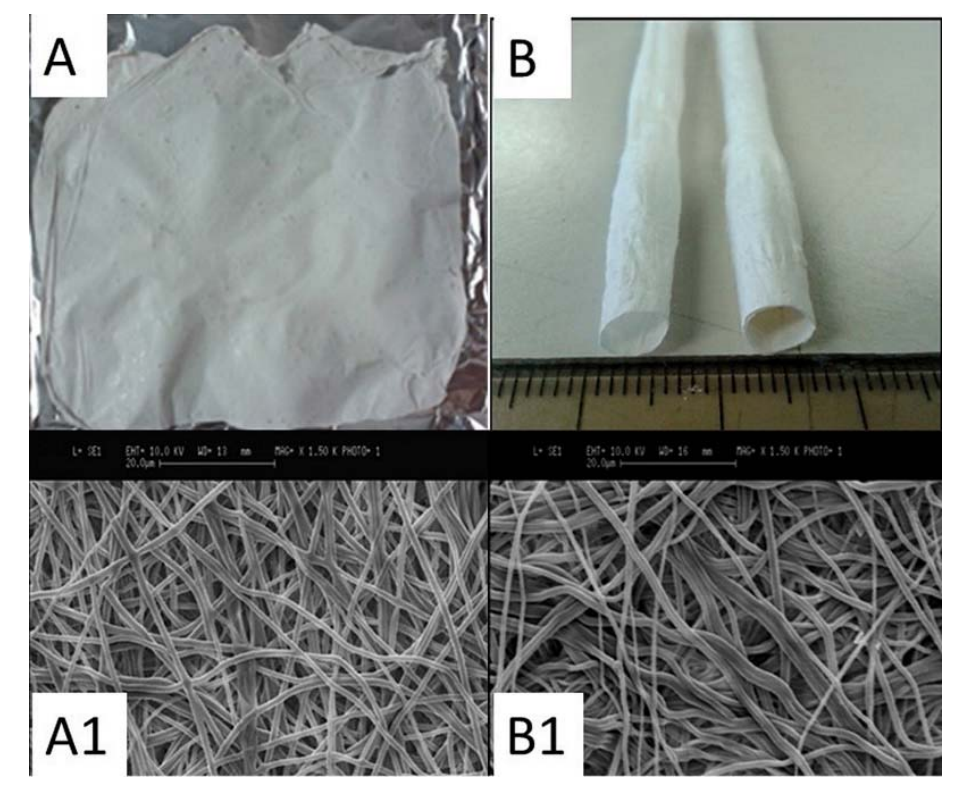

Figure 1. (A): macrograph of 2D ESF mat, (A1): SEM image of its surface (scale bar $=20 \mu \mathrm{m}$ ); (B): macrograph of ESF tubular structures, $\varnothing=6 \mathrm{~mm}$, (B1): SEM image of the external surface of the ESF tubes (scale bar $=20 \mu \mathrm{m}$ ).

The electrospun SF samples were coated with type A gelatin crosslinked in situ by a Michaeltype addition reaction that creates covalent bonds between gelatin amino groups and $\beta$-carbons of $\mathrm{N}-\mathrm{N}^{\prime}$-methylene bis-acrylamide (MBA). The gel is prepared by dissolving $1 \mathrm{~g}$ of gelatin (from porcine skin, G1890 Sigma-Aldrich) in $6 \mathrm{~mL}$ of distilled water at $50{ }^{\circ} \mathrm{C}$, adding $20 \mu \mathrm{L}$ of triethylamine to increase the $\mathrm{pH}$ to 10 and then $\mathrm{MBA}(23,3 \mathrm{mg})$ as crosslinking agent [20]. Completion of the crosslinking reaction requires $24 \mathrm{~h}$ at $50^{\circ} \mathrm{C}$.

To obtain a homogeneous coating on the ESF substrates, different methods were tested. The 2D sample mat was kept taut and suspended on a glass container by fixing the ends with plumbs in turn anchored to the container walls with a stainless steel wire (Figure 2a and $b$ ). To proceed with the coating (Figure 2b), the crosslinking solution was deposited on the ESF mat using a plastic syringe, then placing the system in an oven for $24 \mathrm{~h}$ at $50{ }^{\circ} \mathrm{C}$.

The system specifically set up for 3D ESF substrates (Figure 2c) is composed of a battery motor resistant to high temperatures, which, through a steel shaft and a rubber joint, transmits the rotation to a glass bar on which an electrospun fibroin tube had been previously mounted (Figure 2c). The rotation speed of the system generated by the motor is $5 \mathrm{rpm}$. To ensure a correct transmission of motion from the rotating shaft to the glass bar, couplings between polymeric materials were selected to avoid corrosion and wear problems. The system composed of glass bar, plastic fittings and rotating metal shaft is inserted into a $50 \mathrm{~mL}$ Falcon tube drilled at the ends to allow the connection to the rotating shaft on one side and on the other the insertion in a support for ball bearings used to keep the system in axis without interfering with the rotation (Figure 2c).

Different approaches were investigated for the 3D fibroin structure coating:

I. "static dipping": deposition by contact between the outer surface of the fibroin tube and the solution of gelatin placed in a preheated aluminium container, provided with housings necessary for inserting the ESF tube mounted on the spindle.

II. "dynamic dipping": dipping of the ESF tube kept in motion by the automatic system into the gelatin solution. The gelatin solution is placed in a preheated cylindrical plastic container (Figure 2c), and the ESF tube, preheated and mounted on the system, is coated by dipping inside this container, while it is kept rotating. 
After the gelatin impregnation, to obtain a homogeneous coating in a confined environment, each system is transferred inside the Falcon tube that is sealed at the extremities, and housed in the device, kept in constant rotation and placed in an oven at $50{ }^{\circ} \mathrm{C}$ for 24 hours to allow for the complete gelatin crosslinking.
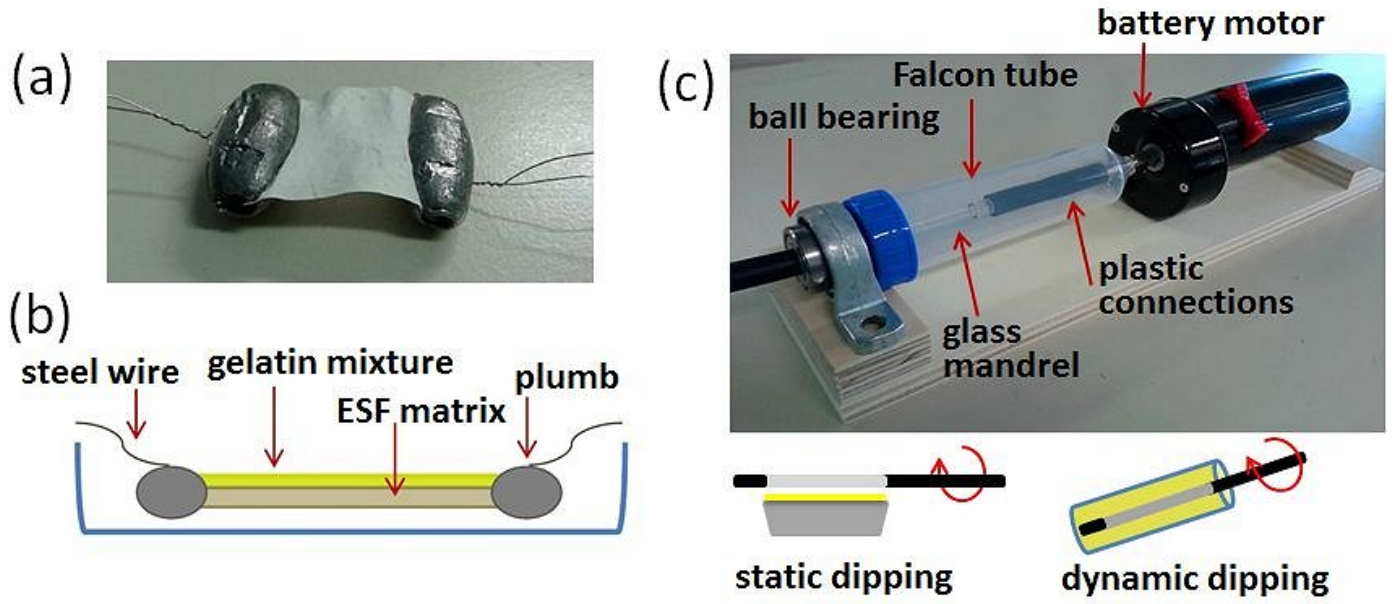

Figure 2. (a) photograph and (b) schematic representation of the anchoring system for coating the ESF mat with gelatin while crosslinking; (c) photograph of the coating device for ESF tubes showing the different components; the drawings below schematically show the two approaches studied for the gelatin coating.

Finally, all gelatin-coated ESF samples were purified by consecutive washing steps with pure ethanol, $\mathrm{HCl} 0.1 \mathrm{M}$ and distilled water to eliminate possible unreacted MBA and low molecular weight substances. The coated samples were then dehydrated by dipping in ethanol solutions and stored at room temperature under reduced pressure until analysis.

\subsection{Characterization of Coated Samples}

The wall thickness of uncoated and coated ESF samples $(n=7)$ after swelling in water was measured with a micrometer (Somet).

Morphology was evaluated by scanning electron microscopy (SEM, StereoScan 360, Cambridge Instruments). The average fiber diameter was calculated from SEM images acquired on five different samples; for each sample 20 measures were acquired with ImageJ v.1.42q software (NIH, Bethesda, MD, USA). For SEM analysis, gelatin-coated samples were dehydrated in graded ethanol solutions, sputter-coated with gold (Edwards Sputter Coater 5150B) and observed with a $10 \mathrm{keV}$ accelerating voltage.

Swelling and weight loss in demineralized water at $37^{\circ} \mathrm{C}$ were evaluated on five specimens per sample type. Wet sample weight at different time points (up to 25 days) was determined by blotting the sample surface with filter paper to remove excess water and then immediately weighing. The weight variation of the samples was calculated following Equation (1):

$$
\Delta w e i g h t \%=\frac{W i-W o}{W o} * 100
$$

where $W i$ is the sample weight at time point $i$ and $W o$ is the initial dry weight of the sample.

Weight variation of the samples for each test time is represented by the average value and standard deviation of data obtained from five specimens of the same type.

Circumferential tensile mechanical tests were carried out on cylindrical specimens ( $5 \mathrm{~mm}$ length segments) of coated and uncoated ESF tubes. The samples ( $n=5$ for each test and material) previously brought to the swelling plateau in distilled water at $37^{\circ} \mathrm{C}$ were housed between two L-shaped stainless steel grips. 
The tests were performed by use of a dynamic-mechanical analyzer (DMA, Q800, TA Instruments), with a preload of $0.005 \mathrm{~N}$ and a force ramp of $0.5 \mathrm{~N} / \mathrm{min}$ up to sample break. From the obtained stress/strain curves, the evaluated parameters were: elastic modulus (E), ultimate tensile stress $(\sigma b)$ and strain at break $(\varepsilon b)$.

\subsection{Cytocompatibility Tests}

For in vitro interaction tests with L929 cells (murine fibroblasts, ECACC $\left.\mathrm{n}^{\circ} 85011425\right)$ circular specimens of gelatin-coated ESF samples punched from the tubular scaffolds $(\varnothing=6 \mathrm{~mm}$, uncoated ones as control) were disinfected with a $70 \%$ ethanol solution under a laminar flow hood for $1 \mathrm{~h}$ and left to dry in sterile conditions for $24 \mathrm{~h}$.

Indirect cytotoxicity tests were carried out to verify the possible release of toxic products and consisted in culturing the cells in the presence of extracts (eluates) of the samples. The eluates were obtained by immersing the samples $(\mathrm{n}=3$, per time point) in complete Dulbecco's Modified Eagle Medium (DMEM, Sigma Aldrich) for 1, 3 and 7 days. L929 cells (density $=1 \times 10^{4}$ cells/well) were seeded in the 96-multiwell culture plastic (TCPS) and cultured for $24 \mathrm{~h}$ to allow for cell adhesion on the well bottom. After that, the medium was replaced with the obtained DMEM eluates, using DMEM only as control. After $24 \mathrm{~h}$ of culture, cell viability was investigated with the MTT colorimetric assay, based on the use of the MTT tetrazolium

salt [3-(4,5-dimethylthiazol-2-yl)-2,5-diphenyl tetrazolium bromide)] that is reduced to a purple insoluble formazan product in living cells by mitochondrial succinate dehydrogenase. The absorbance was evaluated at $570 \mathrm{~nm}$ with a spectrophotometer (TECAN, Genios Plus)

Direct contact tests were performed with Human umbilical cord vein endothelial cells (HUVEC) on crosslinked gelatin samples and gelatin-coated ESF mats (uncoated ones as control). Primary cultures of HUVEC were obtained through enzymatic digestion of the human umbilical cord vein provided by the Cittadella Hospital (Verona) and processed within 24 hours of delivery.

Specimens having a diameter of $6 \mathrm{~mm}$ were obtained with a manual die $(\mathrm{n}=3)$, washed under a laminar flow hood in a 50\% ethanol solution for 2 hours, in pure ethanol for a further 2 hours and then allowed to dry overnight in sterile conditions. Subsequently, the specimens were transferred to 96 well TCPS plates.

$5 \times 10^{3}$ HUVEC per well were seeded on each sample and allowed to adhere and proliferate for up to 7 days under standard tissue culture conditions.

In the case of gelatin gels, at each time-point (1, 3 and 7 days), non-adherent cells were removed by rinsing with PBS. The adherent cell were fixed with $4 \%$ formalin overnight and stained with $0,5 \%$ (w/v in 20\% ethanol) toluidine blue. Cell adhesion on the matrices was qualitatively evaluated by optical microscopy.

Cell proliferation on silk fibroin-based matrices was quantitatively evaluated from the total protein content obtained by lysis of the cells grown for 7 days on them; adherent cells were treated with $0.1 \%$ Triton $\mathrm{X} 100$ overnight at $4{ }^{\circ} \mathrm{C}$ to cause their lysis and the total protein content was determined using BCA Protein Assay. Absorbance was measured at $570 \mathrm{~nm}$ and results expressed in $\mu \mathrm{g} / \mathrm{ml}$, by use of a calibration curve obtained with bovine serum albumin.

\subsection{Statistical Analysis}

Data are presented as mean values \pm standard deviation and statistically compared by two-tailed $\mathrm{t}$-Test. Differences between groups were considered significant for $\mathrm{p} \leq 0.05$.

\section{Results and Discussion}

The morphology of the 2D matrices and electrospun tubular SF structures was analyzed by SEM observation. SEM images show the electrospun structure formed by randomly arranged fibers, typical of non-woven materials (Figure 1 A1 and B1). No presence of defects (beads) due to the electrospinning process can be found. Furthermore, both mats and tubes exhibit a similar morphology of the internal surface, i.e., the surface in contact with the collector, and the external one. 
Fiber dimensions, calculated using ImageJ software, are overall homogeneous with the same matrix (flat or tubular) and surface area considered (internal vs external), with values in the range 400-1200 nm following a bell-shaped distribution, and a mean value of less than $900 \mathrm{~nm}$ (Table 1).

Table 1. Physical properties of 2D and 3D ESF samples, before and after gelatin coating.

\begin{tabular}{|c|c|c|c|c|c|}
\hline \multirow{2}{*}{ Sample } & \multirow{2}{*}{$\begin{array}{c}\text { Fiber } \varnothing(\mathrm{nm}) \\
(\text { Mean Value } \pm \text { sd) }\end{array}$} & \multirow{2}{*}{$\begin{array}{c}\text { Thickness, } \mu \mathrm{m} \\
\text { (Wet Conditions) }\end{array}$} & \multicolumn{3}{|c|}{ Tensile Properties } \\
\hline & & & E (MPa) & $\sigma_{\mathrm{b}}(\mathrm{MPa})$ & $\varepsilon_{\mathrm{b}}(\%)$ \\
\hline ESF mat & $856 \pm 147$ & $122.5 \pm 20.6$ & & & \\
\hline gelatin-coated 2D ESF & & $176.8 \pm 29.7$ & & & \\
\hline ESF tube $\ldots$ & $855 \pm 236$ & $212.5 \pm 43.1$ & $1.82 \pm 0.49$ & $2.41 \pm 0.69$ & $141.53 \pm 24.40$ \\
\hline gelatin-coated 3D ESF & & $306.7 \pm 45.1$ & $0.58 \pm 0.25$ & $1.17 \pm 0.48$ & $152.72 \pm 41.73$ \\
\hline
\end{tabular}

At SEM, it was possible to qualitatively assess the degree of penetration of the cross-linked gelatin gel inside the thickness of the electrospun 2D mat although the gelatin deposition took place only on one of the two surfaces. In the image shown in Figure $3 b$ it is possible, in fact, to observe a homogeneous and compact layer of gelatin on both surfaces. In addition, (Figure 3a) the nanofiber structure was barely distinguishable under the homogeneous coating of the crosslinked gelatin gel. The typical porosity of electrospun structures therefore allows the passage of gelatin through the entire thickness of the 2D matrix.

Consequently, also for the tubular matrix the coating procedure was applied from the external surface, thus obtaining the complete penetration of the crosslinked gel.

As shown in Figure $3 c$ and $d$, in both static and dynamic dipping, on the coated surfaces it is possible to note the presence of gelatin uniformly distributed among the fibers. In particular, after the "static dipping", the nanofiber morphology of the electrospun structure was still evident (Figure 3c), while the "dynamic dipping", qualitatively, produces a coating with a greater thickness of gelatin (Figure 3d). Actually, the fibers are less evident than after the "static" method.

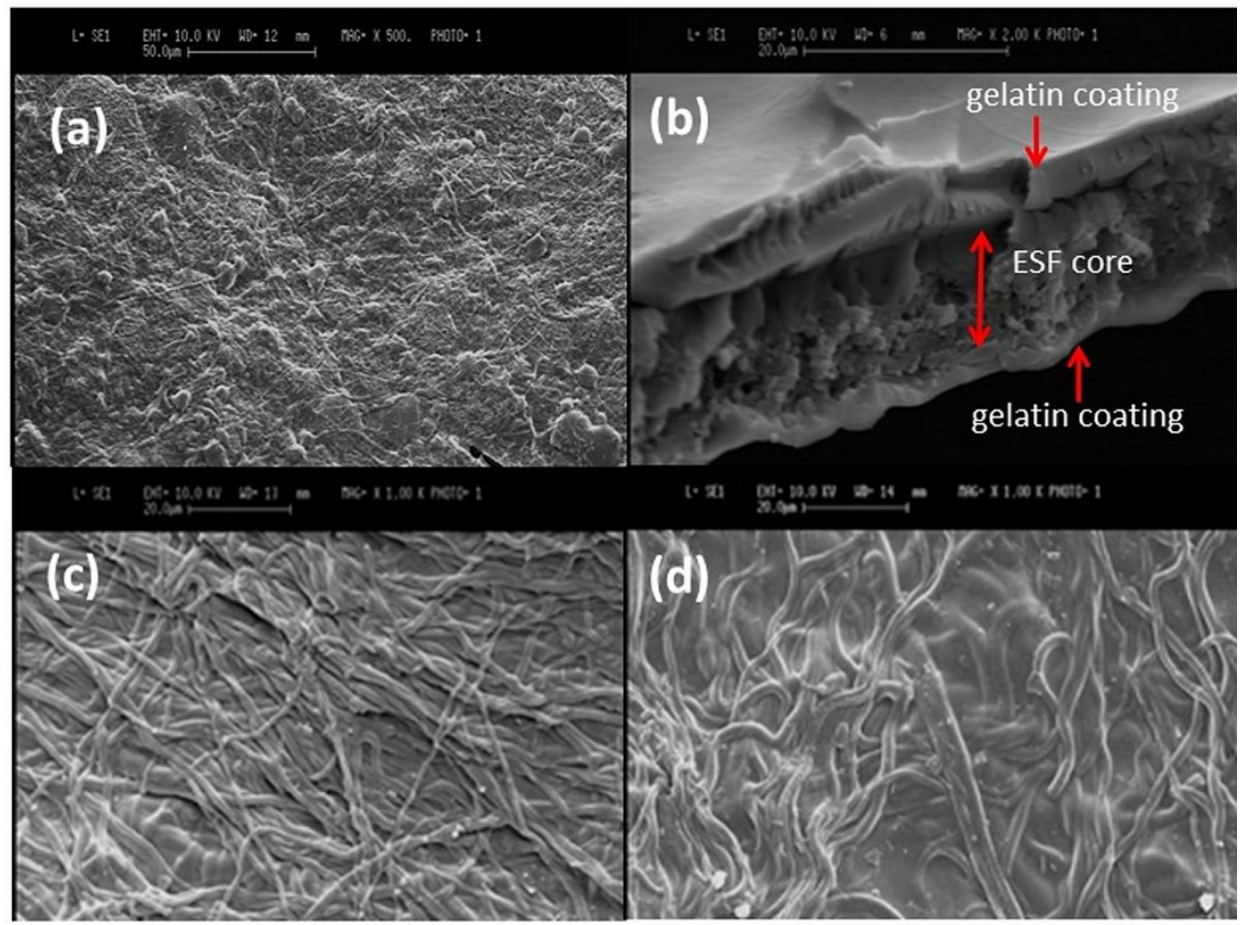

Figure 3. Morphology of gelatin-coated ESF samples. SEM images of (a) surface (scale bar $=50 \mu \mathrm{m})$ and (b) cross-section of the gelatin-coated mat (scale bar $=20 \mu \mathrm{m})$; (c) surface of the ESF tube by "static dipping", (d) surface of the ESF tube by "dynamic dipping" (scale bar $=20 \mu \mathrm{m}$ ). 
As a more efficient method, the "dynamic dipping" was therefore chosen for coating the ESF tubes with cross-linked gelatin.

From the measures performed with the micrometer, a significant difference $(p<0.05)$ was observed between the wall thickness of the ESF tubes and that of the ESF mat, both in the anhydrous state (data not shown) and at the swelling plateau (Table 1). This is attributable to the different surface of the collector $\left(72 \mathrm{~cm}^{2}\right.$ for mats and $18.85 \mathrm{~cm}^{2}$ for tubes) for the same volume of the fibroin solution used for electrospinning.

By comparing the thickness in wet conditions of the coated samples with the uncoated ones (Table 1), it is possible to highlight a statistically significant difference $(p<0.05)$, with an increase of thickness higher that $40 \%$.

Weight variation tests, performed at $37^{\circ} \mathrm{C}$ in water on $5 \mathrm{~mm}$ length tubular specimens indicated for ESF samples a rapid swelling within the first $10 \mathrm{~min}$ of incubation, reaching their maximum water uptake $(\approx 500 \%)$ and maintaining this value of plateau until the 25th day (Figure 4 , red line). The coated samples (ESFgel), instead, show higher swelling values $(p<0.05)$, reaching an increase of $1000 \%$ after 60 minutes; subsequently, the weight variation still increases to $1600 \%$ up to 14 days and then starts decreasing (Figure 4, blue line), probably due to the beginning of the gelatin gel degradation.

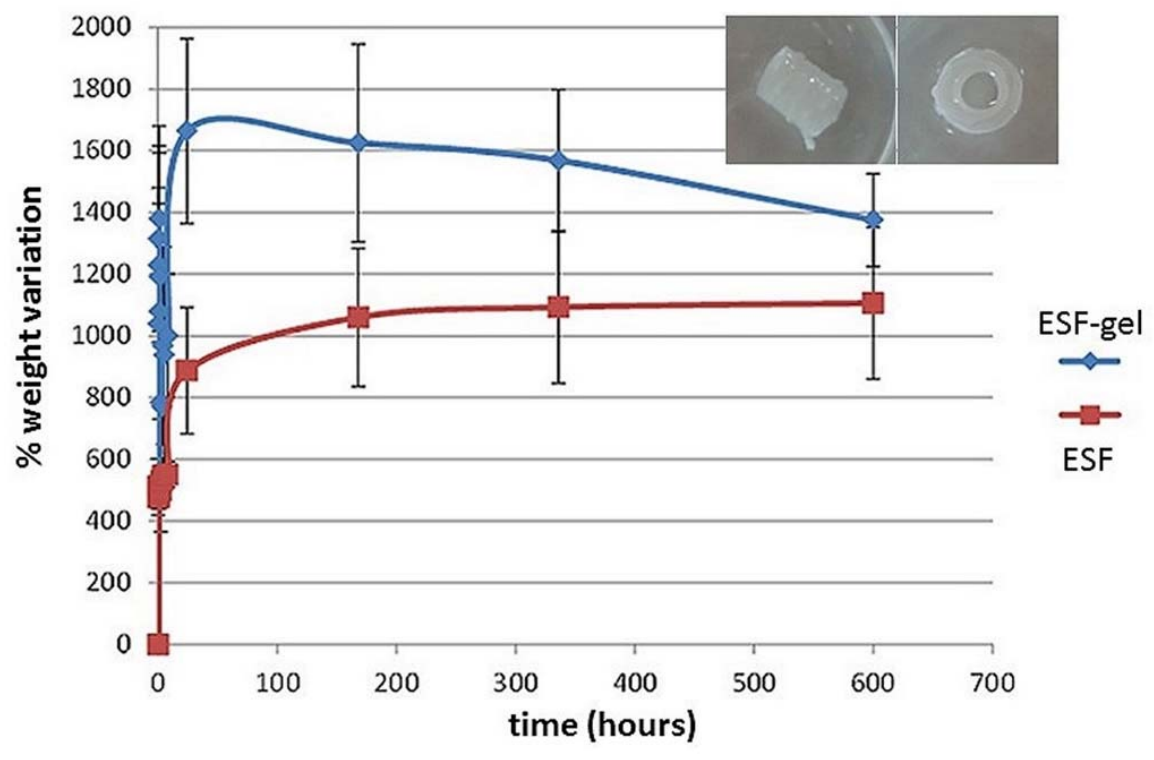

Figure 4. Swelling behavior and weight loss for ESF and ESF-gel coated samples until day 25 (600 hours). On the right upper corner, two images of a swollen ESF-gel sample.

A comparison between circumferential tensile properties of gelatin-coated ESF tube versus non coated samples is provided by representative stress/strain curves shown in Figure 5. The values of elastic modulus (E), stress $(\sigma \mathrm{b})$ and deformation $(\sigma \mathrm{b})$ at break are reported in Table 1.

It is possible to notice how, with the same deformation, the corresponding stress values for ESFgel are lower than those found for uncoaed ESF, indicating a lower stiffness. This observation is confirmed by the values of elastic modulus, significantly different by comparing the gelatin-coated samples with those of fibroin alone $(p<0.05)$.

The lower mechanical characteristics for ESF-gel are probably attributable to the gelatin coating, which balances the greater mechanical properties of fibroin alone. 


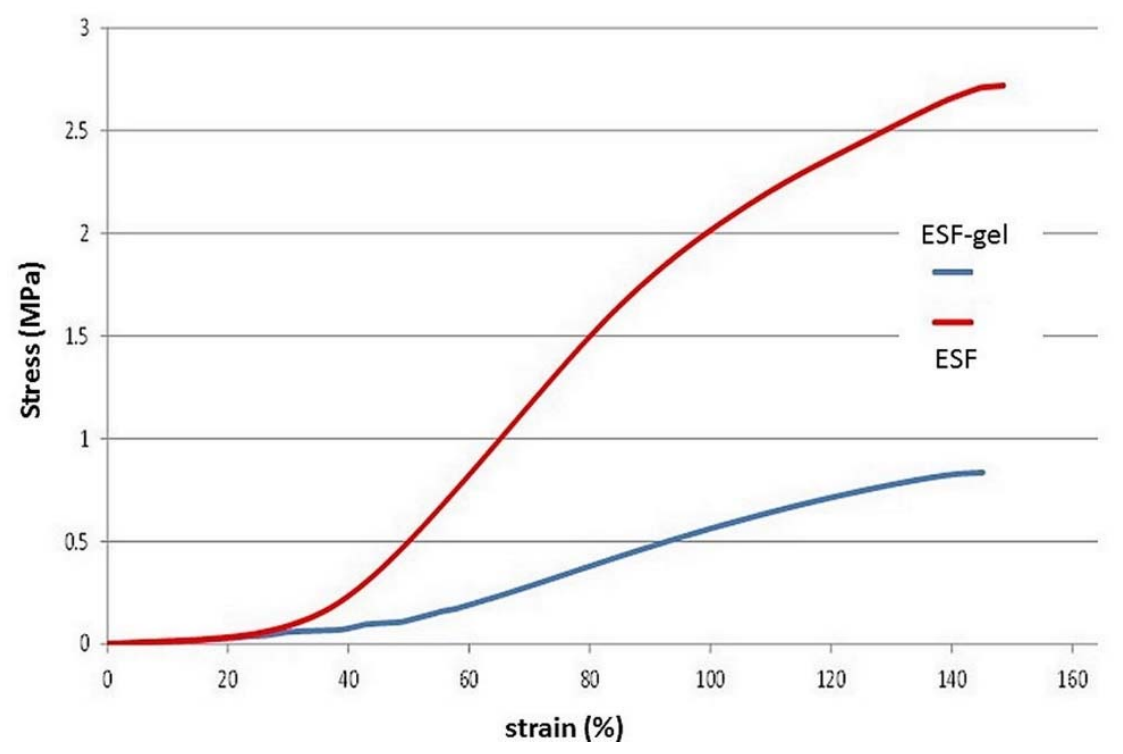

Figure 5. Representative stress/strain curves for gelatin-coated ESF tubular structure (ESF-gel) and uncoated ESF obtained in circumferential tensile tests.

\section{In Vitro Interaction Tests with Cells}

The MTT assay performed after 24 hours of contact of the L929 cells with the culture medium in contact with the samples (Figure 6) showed that, in the one-day eluates, the cell viability levels were significantly higher $(p<0.05)$ for fibroin eluates $(E S F)$ with respect to the control and a viability comparable to the control for ESF-gel eluates. For eluates after 3 days, the same trend was even more evident. At 7 days, however, the viability of the cells in contact with the ESF-gel eluates increased considerably and became comparable $(p>0.05)$ with that of the ESF samples. Both these values were higher than the control $(\mathrm{p}<0.05)$. Controls were represented by DMEM only, left in the incubator for the same periods of time (1, 3 and 7 days).

It is therefore possible to conclude that neither the electrospun fibroin nor the coating obtained by crosslinking gelatin with MBA release toxic substances that could decrease cell viability.

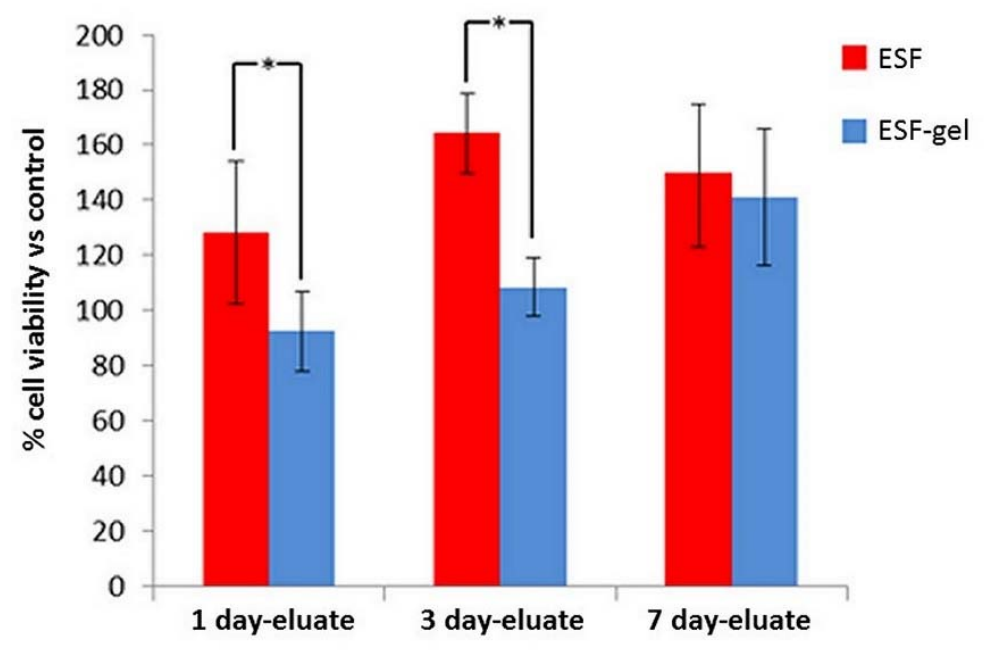

Figure 6. MTT test results for indirect cytotoxicity of ESF and ESF-gel samples incubated for 1-7 days in culture medium, using the bare culture medium maintained in the incubator for the same days as a control. $\left(^{*}\right)$ p-value $<0.05$. 
Direct cytocompatibility results with L929 cells were described in reference [21], where the cells were seeded on samples prepared as for the indirect cytotoxicity tests and cultured for up to 7 days.

Alamar Blue assay indicated a good cytocompatibility for both uncoated and coated ESF samples with a linear increase of cell viability with culture time (1,3 and 7 days), cell viability being comparable to that observed on the culture plastic (TCPS). At SEM, the cells were found spread and flattened already after the first day, and at day 7 their number appeared greater on gelatin-coated samples.

In this work, direct culture tests were performed with primary human endothelial cells (HUVEC). HUVEC were isolated from human umbilical cord vein, processed within 24 hours of delivery, seeded and cultured up to 7 days on both crosslinked gelatin alone and ESF-coated samples.

After the first day of culture on the matrix of gelatin, many viable cells both rounded and adherent were visible; over time, they tended to form organized colonies. Representative OM images are provided in Figure 7, left. Therefore, these results suggest that gelatin crosslinked with MBA represents a favourable environment for endothelial cells.

The evaluation of HUVEC growth on silk fibroin-based supports was carried out by quantitative determination of the total proteins in cell lysates. It can be observed (Figure 7, right) that gelatincoated ES fibroin displayed a higher total protein content than uncoated ES fibroin $(p<0.05)$, therefore indicating a better cell growth on this type of matrix.
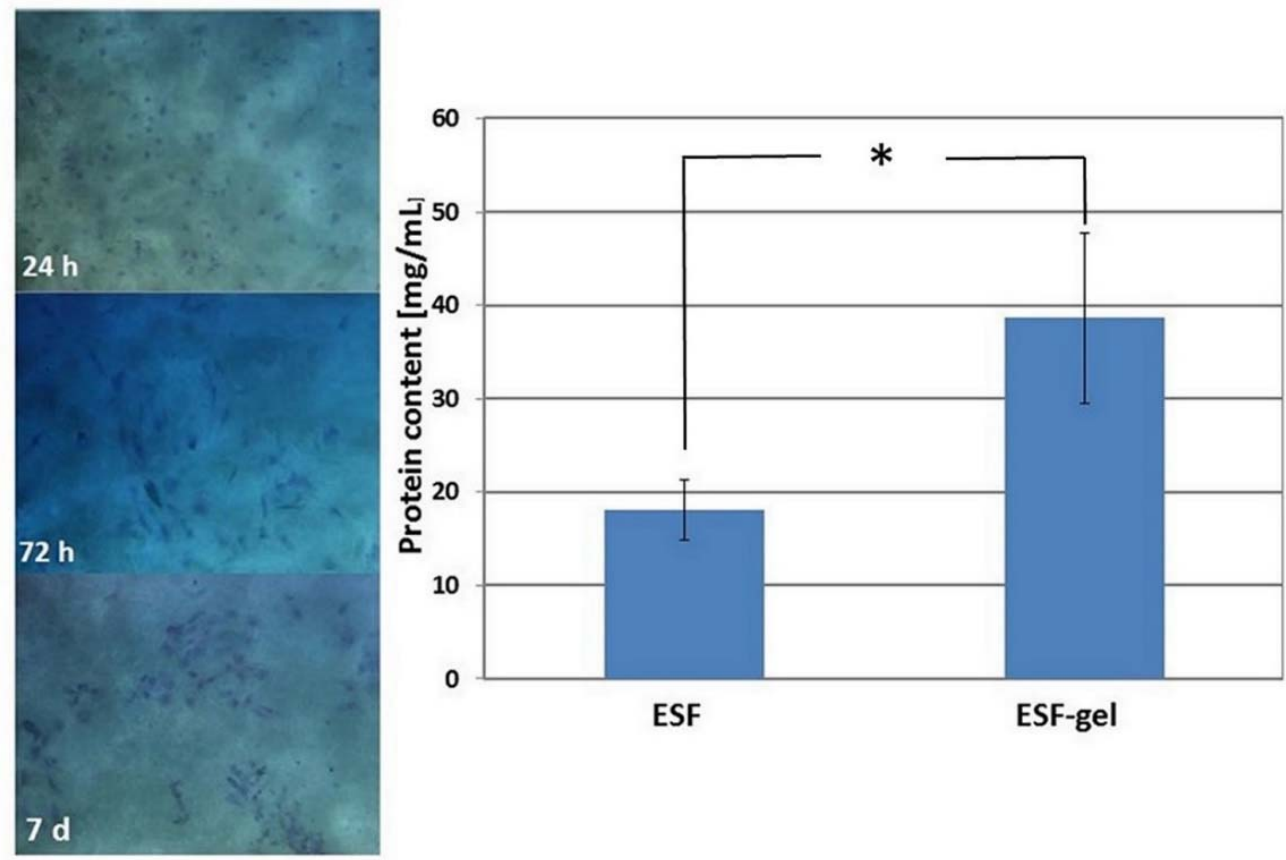

Figure 7. Left: OM images (100 X) of HUVEC cultured on crosslinked gelatin samples $24,72 \mathrm{~h}$ and 7 days after seeding, fixing with formaldehyde and staining with toluidine blue; right: total protein content as evaluated after lysis of HUVEC cultured for 7 days on gelatin-coated (ESF-gel) and uncoated electrospun ESF samples $(n=3) .^{*}=p<0.05$.

\section{Conclusions}

The novel 2D and 3D ESF-gel structures developed in this work demonstrated adequate morphological properties to support cell colonization, combined with good stability in an aqueous environment and acceptable mechanical properties, with an increase in flexibility compared to the structure of electrospun fibroin.

The crosslinking method developed overcomes gelatin limited stability at $37^{\circ} \mathrm{C}$, as the gel coating proved to be stable up to 14 days while maintaining good cell compatibility, which is therefore not significantly affected by the crosslinking reaction. 
Interestingly, when the crosslinking mixture is applied to a substrate containing primary or even secondary amino groups, these groups can participate in the reaction, being incorporated into the gelatin coating, thus increasing the coating stability on the surface.

The per se remarkable biocompatibility of SF is therefore combined with a coating that acts as a sealant and is capable to promote cell adhesion and proliferation

Conflicts of Interest: The authors declare no conflict of interest.

\section{References}

1. Li, W.-J.; Laurencin, C.T.; Caterson, E.J.; Tuan, R.S.; Ko, F.K. Electrospun nanofibrous structure: a novel scaffold for tissue engineering. J. Biomed. Mater. Res. 2002, 60, 613-621, doi:10.1002/jbm.10167.

2. Pham, Q.P.; Sharma, U.; Mikos, A.G. Electrospinning of polymeric nanofibers for tissue engineering applications: A review. Tissue Eng. 2006, 12, 1197-1211.

3. Khorshidi, S.; Solouk, A.; Mirzadeh, H.; Mazinani, S.; Lagarón, J.M.; Sharifi, S.; Ramakrishna, S. A review of key challenges of electrospun scaffolds for tissue-engineering applications. J. Tissue Eng. Regen. Med. 2015, 10, 715-738, doi:10.1002/term.1978.

4. Vepari, C.; Kaplan, D. Silk as a biomaterial. Prog. Polym. Sci. 2007, 32, 991-1007, doi:10.1016/j.progpolymsci.2007.05.013.

5. Altman, G.H.; Diaz, F.; Jakuba, C.; Calabro, T.; Horan, R.L.; Chen, J.; Lu, H.; Richmond, J.; Kaplan, D. Silkbased biomaterials. Biomater. 2003, 24, 401-416, doi:10.1016/s0142-9612(02)00353-8.

6. Chomachayi, M.D.; Solouk, A.; Mirzadeh, H. Electrospun silk-based nanofibrous scaffolds: fiber diameter and oxygen transfer. Prog. Biomater. 2016, 5, 71-80, doi:10.1007/s40204-016-0046-6.

7. Alessandrino, A.; Marelli, B.; Arosio, C.; Farè, S.; Tanzi, M.C.; Freddi, G. Electrospun Silk Fibroin Mats for Tissue Engineering. Eng. Life Sci. 2008, 8, 219-225, doi:10.1002/elsc.200700067.

8. Marelli, B.; Alessandrino, A.; Farè, S.; Freddi, G.; Mantovani, D.; Tanzi, M.C. Compliant electrospun silk fibroin tubes for small vessel bypass grafting. Acta Biomater. 2010, 6, 4019-4026, doi:10.1016/j.actbio.2010.05.008.

9. Valenzuela, F.; Covarrubias, C.; Martínez, C.; Smith, P.; Díaz-Dosque, M.; Yazdani-Pedram, M. Preparation and bioactive properties of novel bone-repair bionanocomposites based on hydroxyapatite and bioactive glass nanoparticles. J. Biomed. Mater. Res. Part B: Appl. Biomater. 2012, 100, 1672-1682, doi:10.1002/jbm.b.32736.

10. Wang, S.; Zhang, Y.; Yin, G.; Wang, H.; Dong, Z. Electrospun polylactide/silk fibroin-gelatin composite tubular scaffolds for small-diameter tissue engineering blood vessels. J. Appl. Polym. Sci. 2009, 113, 26752682, doi:10.1002/app.30346.

11. Correia, T.R.; Ferreira, P.; Vaz, R.; Alves, P.; Figueiredo, M.; Correia, I.J.; Coimbra, P. Development of UV cross-linked gelatin coated electrospun poly(caprolactone) fibrous scaffolds for tissue engineering. Int. J. Boil. Macromol. 2016, 93, 1539-1548, doi:10.1016/j.ijbiomac.2016.05.045.

12. E Ghezzi, C.; Marelli, B.; Muja, N.; Hirota, N.; Martin, J.G.; E Barralet, J.; Alessandrino, A.; Freddi, G.; Nazhat, S.N. Mesenchymal stem cell-seeded multilayered dense collagen-silk fibroin hybrid for tissue engineering applications. Biotechnol. J. 2011, 6, 1198-1207, doi:10.1002/biot.201100127.

13. Marelli, B.; Achilli, M.; Alessandrino, A.; Freddi, G.; Tanzi, M.C.; Farè, S.; Mantovani, D. CollagenReinforced Electrospun Silk Fibroin Tubular Construct as Small Calibre Vascular Graft. Macromol. Biosci. 2012, 12, 1566-1574, doi:10.1002/mabi.201200195.

14. Casper, C.L.; Yamaguchi, N.; Kiick, K.L.; Rabolt, J.F. Functionalizing Electrospun Fibers with Biologically Relevant Macromolecules. Biomacromolecules 2005, 6, 1998-2007, doi:10.1021/bm050007e.

15. Truong, Y.B.; Glattauer, V.; Briggs, K.L.; Zappe, S.; Ramshaw, J.A.M. Collagen-based layer-by-layer coating on electrospun polymer scaffolds. Biomater. 2012, 33, 9198-9204, doi:10.1016/j.biomaterials.2012.09.012.

16. Lee, K.Y.; Mooney, D.J. Hydrogels for Tissue Engineering. Chem. Rev. 2001, 101, 1869-1880, doi:10.1021/cr000108x.

17. Mohammadzadehmoghadam, S.; Dong, Y. Fabrication and Characterization of Electrospun Silk Fibroin/Gelatin Scaffolds Crosslinked with Glutaraldehyde Vapor. Front. Mater. 2019, 6, doi:10.3389/fmats.2019.00091. 
18. De-bing, S.; Zhi-hui, D.; Wei-guo, F. Study on the properties of the electrospun silk fibroin/gelatin blend nanofibers for scaffolds. J. Appl. Polym. Sci. 2009, 111, 1471-1477.

19. Okhawilai, M.; Rangkupan, R.; Kanokpanont, S.; Damrongsakkul, S. Preparation of Thai silk fibroin/gelatin electrospun fiber mats for controlled release applications. Int. J. Boil. Macromol. 2010, 46, 544-550, doi:10.1016/j.ijbiomac.2010.02.008.

20. Negrini, N.C.; Tarsini, P.; Tanzi, M.C.; Farè, S. Chemically crosslinked gelatin hydrogels as scaffolding materials for adipose tissue engineering. J. Appl. Polym. Sci. 2018, 136, 136, doi:10.1002/app.47104.

21. Marcolin, C.; Draghi, L.; Tanzi, M.C.; Farè, S. Electrospun silk fibroin-gelatin composite tubular matrices as scaffolds for small diameter blood vessel regeneration. J. Mater. Sci. Mater. Electron. 2017, 28, 80, doi:10.1007/s10856-017-5884-9.

(C) 2020 by the authors. Submitted for possible open access publication under the terms and conditions of the Creative Commons Attribution (CC BY) license (http://creativecommons.org/licenses/by/4.0/). 